\title{
A fiberoptic orotracheal intubation successfully performed using a modified Guedel airway in a sedated emergency patient - A case report -
}

Received April 27, 2020

Revised July 7, 2020

Accepted July 7, 2020

\section{Jaeyoung Yang, Seonjin Kim, Bousung Lee, Kwanghaeng Lee, Dongseok Kim, Jaedo Lee, Hee Jung Jun, Jin Sun Yoon, and Sam Soon Cho}

Department of Anesthesiology and Pain Medicine, VHS Medical Center, Seoul, Korea

\author{
Corresponding author \\ Sam Soon Cho, M.D. \\ Department of Anesthesiology and \\ Pain Medicine, VHS Medical Center, \\ 53, Jinhwangdo-ro 61-gil, Gangdong- \\ gu, Seoul 05368, Korea \\ Tel: 82-2-2225-1473 \\ Fax: 82-2-2225-1946 \\ E-mail: chss1756@naver.com
}

\begin{abstract}
Background: Fiberoptic intubation is a powerful and safe technique to deal with airway difficulty, but it requires a lot of training to be able to perform correctly. There are various specialized oral airways for fiberoptic intubation, but none of them have perfect functionality.

Case: A 75-year-old male (body weight $71.6 \mathrm{~kg}$, height $159.3 \mathrm{~cm}$, body mass index 28.22 $\mathrm{kg} / \mathrm{m}^{2}$ ) was diagnosed with acute appendicitis, and it was decided to do a laparoscopic appendectomy. After the induction of general anesthesia, it was impossible to insert the direct laryngoscope deep enough for vocal cord visualization without damaging the teeth because of limited mouth opening. We successfully performed fiberoptic intubation with a newly modified Guedel airway via a longitudinal channel on the convex side and a distal opened lingual end.
\end{abstract}

Conclusions: Our modified Guedel airway can be useful in assisting fiberoptic intubation in unexpectedly difficult airway situations.

Keywords: Airway management; Aneshthesi general; Deep sedation; Intratracheal; Intubation.
Airway assessment is undoubtedly one of the most important tasks in anesthesia. It is impossible to evaluate the airway with just one method. Airway assessments consist of multiple components. Careful history taking and airway examination are essential to find potential airway difficulties. Supplementary methods, such as a plain radiograph or magnetic resonance imaging, can be helpful in accurately assessing the airway.

It is usually best to assess the airway before doing an elective operation, but with emergency operations, proper airway assessment is often difficult. The LEMON (Look externally, Evaluate, Mallampati scoring, Obstruction, Neck mobility) method has been suggested for predicting airway difficulties for trauma patients who undergo emergency surgery [1]. In emergency operations, airway experts who have received specialized training in difficult airway management should be available nearby.

Awake fiberoptic intubation is a powerful and safe technique in managing airway difficulty, but it requires a lot of training to be able to perform it correctly. It has been reported that the success rate on the first attempt of fiberoptic intubation is only $58 \%$, because of the difficulty in the proper positioning of the scope or in advancing the endotracheal tube into the trachea [2]. There are various spe-

This is an Open Access article distributed under the terms of the Creative Commons Attribution Non-Commercial License (http://creativecommons.org/licenses/by-nc/4.0) which permits unrestricted non-commercial use, distribution, and reproduction in any medium, provided the original work is properly cited.

Copyright (C) the Korean Society of Anesthesiologists, 2020 
cialized oral airways for doing fiberoptic intubation, but none of them have perfect functionality.

In this case, we report encountering an unexpectedly difficult airway with a limited mouth opening in an emergency operation, but we successfully performed a fiberoptic intubation in this sedated patient by using our newly modified Guedel airway. It had a longitudinal channel on the convex side, with a distal opening in the lingual end, made by using a conventional Guedel airway that is commonly available in many hospitals.

\section{CASE REPORT}

A 75-year-old male (body weight $71.6 \mathrm{~kg}$, height $159.3 \mathrm{~cm}$, body mass index [BMI] $28.22 \mathrm{~kg} / \mathrm{m}^{2}$ ) came to emergency room with right lower quadrant pain that had started 2 days previously. We diagnosed him with acute appendicitis and decided to do a laparoscopic appendectomy. He had undergone a radical prostatectomy five years previously for prostate cancer. The patient took oral medications for hypertension and Parkinson's disease. His neck was a little bit rigid. The thyromental distance was $7 \mathrm{~cm}$. His mouth opening was 2.5 fingerbreadths. The Mallampati score was a class III. There were no loose, chipped, or damaged teeth. Although there was a concern about airway difficulties on physical examination, we decided to proceed with general anesthesia because there had been no airway issues in previous surgeries.

After giving consent for general anesthesia, the patient entered the operating room. The patient's vital signs were stable prior to general anesthesia. We applied standard monitoring, including electrocardiogram, pulse oximetry, and noninvasive blood measurement. The patient was preoxygenated with $4 \mathrm{~L} / \mathrm{min}$ oxygen via facial mask. General anesthesia was induced with propofol $120 \mathrm{mg}$ and rocuronium $50 \mathrm{mg}$. We inserted the oral airway into the oral cavity. There was no difficulty in mask ventilation. We attempted direct laryngoscopy to visualize the patient's larynx. But after several attempts, it turned out that it was impossible to insert the direct laryngoscope deep enough for vocal cord visualization without damaging his teeth because of a limited mouth opening.

We decided to do a fiberoptic intubation. We expected the laryngeal view on fiberoptic intubation to be bad because the patient had a high BMI $\left(28 \mathrm{~kg} / \mathrm{m}^{2}\right)$ and was fully relaxed. We decided to use the modified Guedel airway that we had made earlier for difficult fiberoptic intubations.

We modified the regular Guedel airway by making a longitudinal channel at the convex side to facilitate airway re- moval after intubation and opened the distal lingual end for a better laryngeal view (Fig. 1).

We inserted the sterilized modified Guedel airway into the mouth. We inserted a fiberoptic bronchoscope $(5.2 \mathrm{~mm}$ diameter, Olympus LF-TP, Japan) into the lubricated 7.5mm endotracheal tube. An assistant lifted the jaw for a better laryngeal view. We advanced the fiberoptic cable through the modified Guedel airway until the glottis was visualized. The fiberoptic cable advanced through the vocal cord until the carina was seen (Fig. 2). The airway was removed gently from the oral cavity. The endotracheal tube was inserted into the trachea along the fiberoptic cable.

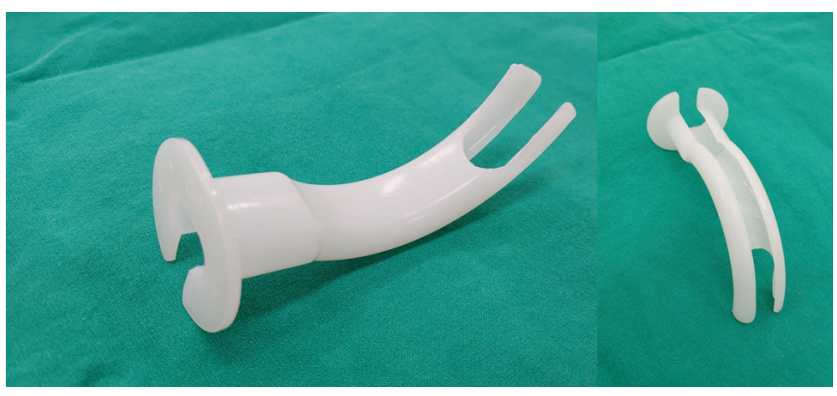

Fig. 1. Photos of modified Guedel airway.

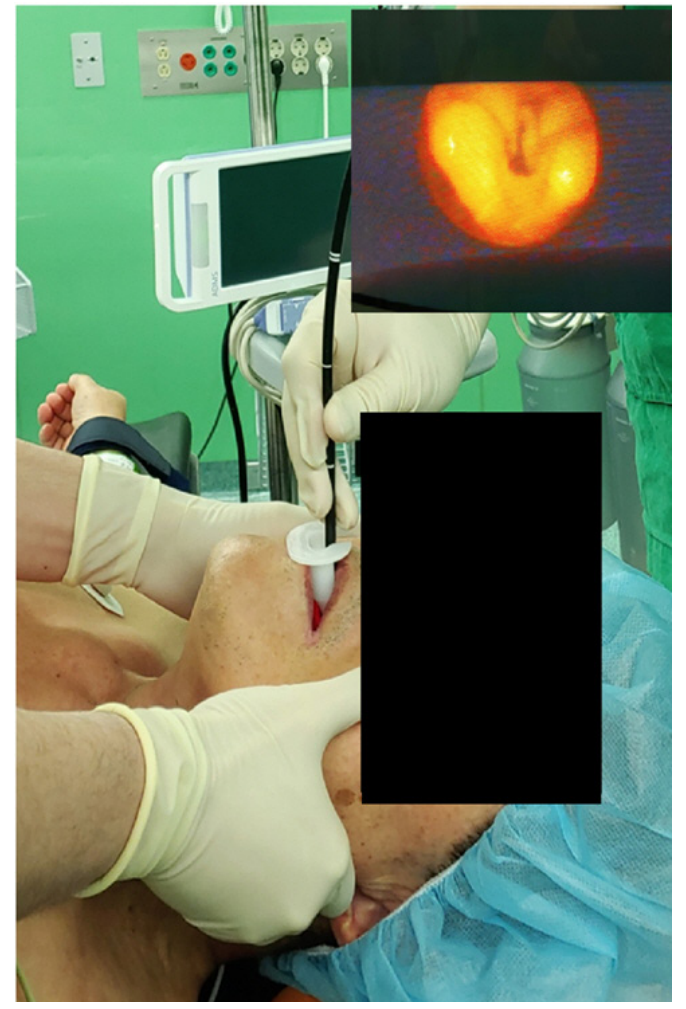

Fig. 2. The fiberoptic intubation procedure with a modified Guedel airway (inset photo: laryngeal view of bronchoscope during the procedure). 
We completed the laparoscopic appendectomy under general anesthesia without any problems. Following surgery, we infused $200 \mathrm{mg}$ of sugammadex via the intravenous route after we had confirmed four twitches in a trainof-four stimulation. We performed extubation when the patient was fully awake. No specific event was reported after extubation.

After the operation, the patient provided written informed consent to have his case details and the accompanying images published.

\section{DISCUSSION}

A careful airway assessment is the most important thing in managing airway difficulties. The failure to identify a potential difficulty in the airway or poor judgement in management planning can lead a poor outcome.

Even if the airway is assessed to be normal following a modified Mallampati score, it is often later found that a patient has a difficult airway. It has been reported that there is a low correlation between a clinical airway assessment and a laryngoscopic grade [3]. Anesthesiologists should always be prepared for unexpected airway difficulties. Various techniques have been developed for difficult airway management, such as supraglottic airway devices, video laryngoscope, lighted stylet, and awake fiberoptic intubation [4].

Supraglottic airway devices, such as a laryngeal mask airway, could be tried in such a situation. Even though there have been many reports that the use of a supraglottic airway device is safe in laparoscopic surgery, it is still controversial among many anesthesiologists. Supraglottic airway devices may not offer perfect airway protection from regurgitated gastric contents in surgeries involving pneumoperitoneum [5].

In a meta-analysis comparison between video laryngoscopy and fiberoptic bronchoscopy, there was no significant difference between the failure rate, the first-attempt success rate, and adverse events. Video laryngoscopy was only associated with a shorter intubation time [6]. But video laryngoscopy has a potential limitation with inserting the blade into the airway of those patients with a limited mouth opening [7].

Awake fiberoptic intubation is considered to be the technique of choice for difficult intubation [4].

There are two major obstacles in performing fiberoptic intubation: It can be difficult to get a clear laryngeal view and to advance the tracheal tube. To achieve a clear laryn- geal view, several tips are recommended, such as jaw thrust, oral airway placement, and neck extension [8]. If the endotracheal tube becomes stuck in the glottis, and advancement become difficult, turning the endotracheal tube 90 degrees counterclockwise can be useful in advancing it smoothly [9].

It is more difficult to perform fiberoptic intubation in a sedated patient than in an awake patient, because with the loss of pharyngeal muscle tone in a sedated patient, it is not easy to achieve a clear laryngeal view [10].

Several types of specialized airways for fiberoptic intubation have been developed so far, Ovassapian, Berman, and Williams airways are the most widely used. An Ovassapian airway is almost just like a flat plastic blade with a short channel at the back of the airway for the bronchoscope. It is notorious for its bad laryngeal view.

The Berman airway has a longitudinal split to use for removing it from the bronchoscope. It has a channel for bronchoscopic passage, but there have been complaints about a bad laryngeal view because of its protruding blade tip end, and it easily becomes stuck at the tongue or esophagus [11].

The Williams airway is shorter and has an open distal lingual end. This Williams airway characteristic can provide a better laryngeal view for fiberoptic intubation. But the endotracheal tube can only pass through the inside of the airway, which can limit the size of available endotracheal tubes. It is impossible to remove the airway until the bronchoscope has been extracted [8].

Greenland et al. [8] insisted that the Williams airway is the best among the three of them, because of its best laryngeal view. But there is no perfect airway for fiberoptic intubation so far.

Most of all, these airways are not easy to get in South Korea. There were several efforts to modify the conventional Guedel airway to help with fiberoptic intubation. Lee made an anterior longitudinal channel at the lingual concave side of Guedel airway. It looks like a Patil-Syracuse airway [12]. The anterior longitudinal channel of the Guedel airway was useful in maintaining the fiberoptic cable in the midline of the oral cavity and in removing the airway before endotracheal tube insertion [13]. Choi et al. [14] reported that they performed successful awake fiberoptic orotracheal intubation by using a modified Guedel airway divided in the midline and fixed with silicone tape.

We tried the Lee fiberoptic intubation airway for awake fiberoptic intubation. It had to be removed to the hard pal- 
ate side because it had a longitudinal channel on the concave side of Guedel airway. There was a risk that the fiberoptic cable would be touched and fall out of the trachea when removing the airway for tracheal intubation because of the limited space (Fig. 3A).

We decided to modify the conventional Guedel airway to allow for better fiberoptic intubation. To overcome the shortcomings of the Lee fiberoptic intubation airway, we made a longitudinal channel at the convex side of Guedel airway (Sewoon Medical Co., Korea), so that it can be removed to the tongue side. (Figs. 3B, 4E) The longitudinal channel was made with a boxcutter and smoothened with fine sandpaper. This feature allows for the airway to be removed more safely. This kind of modification was reported by Rastogi et al. [15]. In addition to that, a distally opened lingual end, adapted from the Williams airway, was added to our new design. This can allow us to get a better laryngeal view and more space for manipulating the tip of fiberoptic cable (Fig. 4D). We performed a 3D scan of the modified Guedel airway to show the modification dimensions in detail (Drake 3D Scanner, THOR3D, Russia) (Fig. 4).

Nasotracheal intubation could be a possible option in cases of difficulty with mouth opening, but we did not try that, because it needs a special nasal tube, and epistaxis, which is a common complication of nasotracheal intubation, can cause a difficult laryngeal view on intubation.

No matter how diligently an airway assessment is done, unexpected airway difficulties can occur at any time, especially in emergency surgery situations. If we can prepare a good airway for fiberoptic intubation, it should be useful for safer anesthesia. Our modified Guedel airway can be the good alternative to other airways in terms of accessibil- ity and performance for doing difficult endotracheal intubations. The only product needed to make it is a conventional Guedel airway. Our new airway has all the necessary fiberoptic intubation features, such as a guiding channel for the fiberoptic cable and a distal opened end to allow for
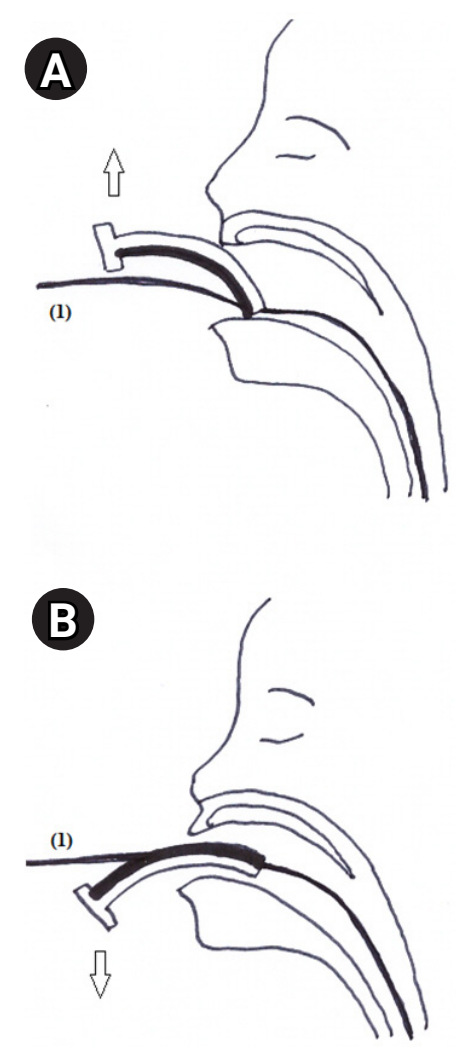

Fig. 3. The scheme of how the modified Guedel airway can be removed from the oral cavity (A: the modified Guedel airway with Lee style, B: our new design). (1): Fiberoptic bronchoscope. Each arrow: direction of oral airway removal. Thick line: opening of the airway for removal.

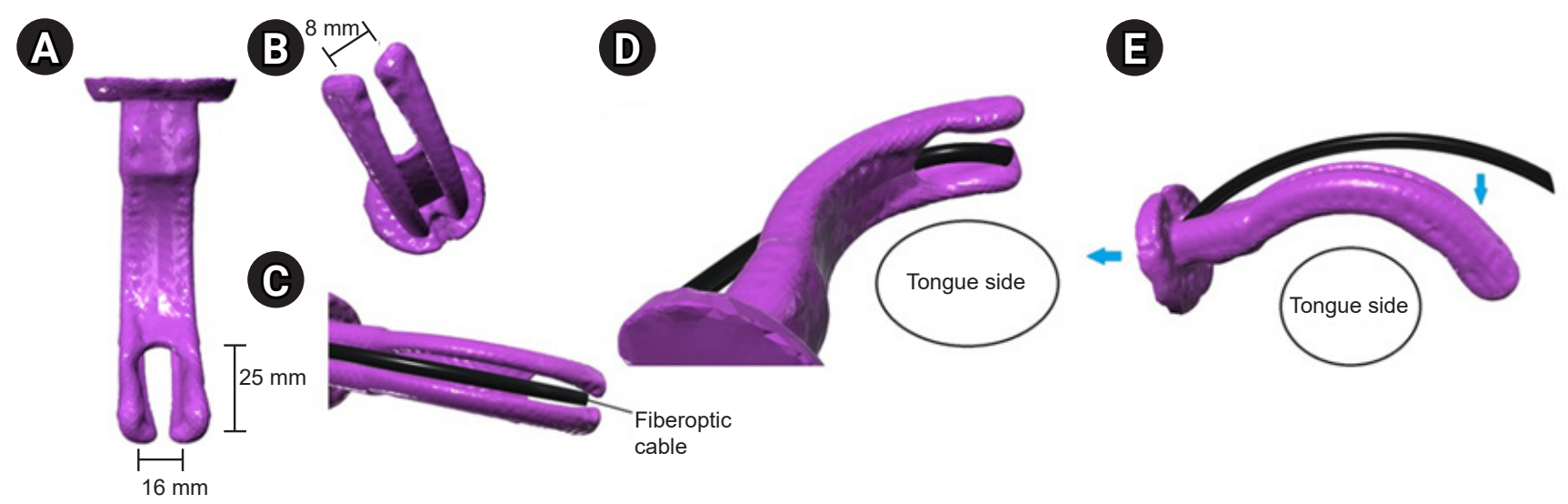

Fig. 4. The 3D scanned images of modified Guedel airway: (A) Front view, (B) Bottom view, (C) The simulation of fiberoptic cable passage along the posterior channel, (D) The distal lingual opening for the flexion of fiberoptic tip, (E) The simulation of the removal of the modified Guedel airway from the fiberoptic cable through the posterior longitudinal channel. 
manipulation of fiberoptic tip.

In conclusion, we report using a modified Guedel airway on a patient with a limited mouth opening capability. This airway has its longitudinal channel on the convex side and a distal opened lingual end. Although further study is necessary to evaluate the utility of our new airway, it could be useful in doing a fiberoptic intubation in an unexpectedly difficult airway situation.

\section{ACKNOWLEDGEMENTS}

Thanks to Min Jo (Prosthetics and Orthotics Center, VHS Medical Center, Seoul, Korea) for his 3D scanning of the modified Guedel airway.

\section{CONFLICTS OF INTEREST}

No potential conflict of interest relevant to this article was reported.

\section{AUTHOR CONTRIBUTIONS}

Writing - original draft: Jaeyoung Yang, Seonjin Kim. Data acquisition: Bousung Lee, Kwanghaeng Lee, Dongseok Kim. Supervision and review: Jaedo Lee, Hee Jung Jun, Jin Sun Yoon, Sam Soon Cho.

\section{ORCID}

Jaeyoung Yang, https://orcid.org/0000-0002-9923-6712

Seonjin Kim, https://orcid.org/0000-0002-6014-2911

Bousung Lee, https://orcid.org/0000-0001-5661-0922

Kwanghaeng Lee, https://orcid.org/0000-0003-1195-3184

Dongseok Kim, https://orcid.org/0000-0002-9876-3393

Jaedo Lee, https://orcid.org/0000-0002-9416-5716

Hee Jung Jun, https://orcid.org/0000-0003-2439-2654

Jin Sun Yoon, https://orcid.org/0000-0002-7133-1814

Sam Soon Cho, https://orcid.org/0000-0003-4039-8140

\section{REFERENCES}

1. Ji SM, Moon EJ, Kim TJ, Yi JW, Seo H, Lee BJ. Correlation between modified LEMON score and intubation difficulty in adult trauma patients undergoing emergency surgery. World J
Emerg Surg 2018; 13: 33.

2. Hanna SF, Mikat-Stevens M, Loo J, Uppal R, Jellish WS, Adams M. Awake tracheal intubation in anticipated difficult airways: LMA Fastrach vs flexible bronchoscope: a pilot study. J Clin Anesth 2017; 37: 31-7.

3. el-Ganzouri AR, McCarthy RJ, Tuman KJ, Tanck EN, Ivankovich AD. Preoperative airway assessment: predictive value of a multivariate risk index. Anesth Analg 1996; 82: 1197-204.

4. Shirgoska B, Netkovski J. New techniques and devices for difficult airway management. Acta Clin Croat 2012; 51: 457-61.

5. Beleña JM, Ochoa EJ, Núñez M, Gilsanz C, Vidal A. Role of laryngeal mask airway in laparoscopic cholecystectomy. World J Gastrointest Surg 2015; 7: 319-25.

6. Alhomary M, Ramadan E, Curran E, Walsh SR. Videolaryngoscopy vs. fibreoptic bronchoscopy for awake tracheal intubation: a systematic review and meta-analysis. Anaesthesia 2018; 73: 1151-61.

7. Jarvi K, Hillermann C, Danha R, Mendonca C. Awake intubation with the Pentax airway scope. Anaesthesia 2011; 66: 314.

8. Greenland KB, Ha ID, Irwin MG. Comparison of the Berman intubating airway and the Williams airway intubator for fibreoptic orotracheal intubation in anaesthetised patients. Anaesthesia 2006; 61: 678-84.

9. Schwartz D, Johnson C, Roberts J. A maneuver to facilitate flexible fiberoptic intubation. Anesthesiology 1989; 71: 470-1.

10. Liu HH, Zhou T, Wei JQ, Ma WH. Comparison between remifentanil and dexmedetomidine for sedation during modified awake fiberoptic intubation. Exp Ther Med 2015; 9: 125964 .

11. Randell T, Valli H, Hakala P. Comparison between the Ovassapian intubating airway and the Berman intubating airway in fibreoptic intubation. Eur J Anaesthesiol 1997; 14: 380-4.

12. Walsh ME, Shorten GD. Preparing to perform an awake fiberoptic intubation. Yale J Biol Med 1998; 71: 537-49.

13. Lee SK, Doo AR. Lee fiberoptic intubating airway for facilitating orotracheal fiberoptic intubation. Korean J Anesthesiol 2013; 65: 368-9.

14. Choi Y, Woo S, Lee JH. Awake fiberoptic orotracheal intubation using a modified Guedel airway in a patient with craniocervical instability and an anticipated difficult airway - a case report -. Anesth Pain Med 2018; 13: 383-7.

15. Rastogi A, Jain A, Singh S, Gyanesh P. Modified Guedel's airway for facilitation of fiberoptic laryngoscopy. J Anaesthesiol Clin Pharmacol 2012; 28: 542-3. 